\title{
Wearable Device for Evaluating the Effect of Training on the Performance of College Baseball Players and Indicator Construction
}

\author{
Shih-Fu Sung, ${ }^{1}$ Yue-Yang Chen, ${ }^{2 *}$ Yung-Ching Chou, ${ }^{3}$ \\ Tsai-Pei Liu, ${ }^{4}$ and Hsien-Hung Chen ${ }^{5}$ \\ ${ }^{1}$ Department of Information Management, I-Shou University, \\ No. 1, Sec. 1, Syuecheng Rd., Dashu District, Kaohsiung City 84001, Taiwan \\ ${ }^{2}$ Department of Business Administration, I-Shou University, \\ No. 1, Sec. 1, Syuecheng Rd., Dashu District, Kaohsiung City 84001, Taiwan \\ ${ }^{3}$ Department of Information Engineering, I-Shou University, \\ No. 1, Sec. 1, Syuecheng Rd., Dashu District, Kaohsiung City 84001, Taiwan \\ ${ }^{4}$ Department of Distribution Management, National Taichung University of Science and Technology \\ No. 129, Sec. 3, Sanmin Rd, North District, Taichung City 404, Taiwan \\ ${ }^{5}$ General Education Center, CTBC Business School, \\ No. 600, Sec. 3, Taijiang Blvd., Annan District, Tainan city 709, Taiwan
}

(Received May 29, 2019; accepted December 14, 2020)

Keywords: wearable device, physical fitness, psychological state, training environment, performance indicator, competition event performance

Baseball has gained popularity at Taiwanese colleges over the last twenty years. The quality of coaching and training is the key factor in the performance of young players during competition. Wireless sensor network (WSN) technology has been developed to index, navigate, store, and analyze data in sports. We investigated the correlations between the performance of college baseball players during a competition game and the following three factors: (1) athletes' physical fitness, (2) athletes' psychological state, and (3) the training environment. Wireless wearable devices were applied in this research to construct the indicators for monitoring the development of players in terms of the three aforementioned factors during routine training. The statistics software SPSS 20.0 was employed to test the correlations between the factors raised in this present research. The findings showed (1) correlations among the physical fitness, psychological state, and training environment and (2) correlations between the effect of training on the competition event performance of baseball players during a competition event and the physical fitness, psychological state, and training environment. In the future, the above indicators can be applied to wearable sensing devices to enhance the effect of training on the performance of the players.

\section{Introduction}

The sport of baseball has been professionalized recently, which has increased the intensity of competition and attracted more spectators. On the other hand, the problem of increased *Corresponding author: e-mail: chenray60@gmail.com https://doi.org/10.18494/SAM.2021.2483 
possibility of injury must be addressed by athletes as well as team managers. For professional athletes, the foremost priority is to stay healthy and prevent sport injuries. ${ }^{(1)}$

Chen et al. ${ }^{(2)}$ pointed out the hurdles in developing baseball in Taiwan. They suggested that a joint effort among different organizations regarding five directions, 'Resources', 'Support', 'Collaboration', 'Integration', and 'Control', will further the development of Taiwanese baseball. For young college baseball athletes, their physical and psychological states during a competition event and a training session are equally crucial to the final result of the competition.

This research is aimed at establishing the correlations among the physical fitness, psychological state, training environment, and training effect and result. Some previous related research has been examined: the results led to a recommendation aimed at improving the quality of training and the result of competition events by paying attention to the needs in terms of the physical fitness, psychological state, and training environment. The objective of the research is twofold: (1) clarify whether there are significant differences among the proposed factors, namely, the physical fitness, psychological state, and training environment, and (2) examine the correlation between the result of the competition event and the physical fitness, psychological state, and training environment.

\section{Theoretical Background}

Physical factors refer to the player's speed, strength, endurance, agility, and flexibility during the sport. Several countries emphasize sport techniques, competition tactics, physical capability, and psychological state during athletes' training sessions and sports research. On the other hand, in addition to the tactics for achieving the ideal performance during the competition, Taiwanese researchers also turn an eye to underscore the career switching by athletes to shed light on the significance of proper protection during training sessions. Hence, the importance of the training environment and scientific training is highlighted in this research, along with the physical fitness and psychological state.

\subsection{Physical fitness of baseball athletes}

The result of training is cumulative over time. The development of the sport of baseball in Taiwan is advocated by the government and local organizations, which mainly target worldwide competition events and professional baseball league games. As baseball gains increasing popularity in Taiwan, problems emerge among team organization, team management, coaching, and the organization of competition events. The future development of baseball is dependent on the problem solving skill regarding the aforementioned problems.

Physical fitness is the premier concern foundation for a baseball athlete, and hence its maintenance and improvement are essential. The coach's 'physical training concept' could directly impact the development of a player in different stages. Sports injury is unavoidable during competitions. Nevertheless, sports injury can be substantially reduced through scientific training and the prevention of excessive training. Efficient training can enhance the physical 
fitness of the player, who, in turn, can adapt to a higher level of training and attain a better performance during competition events. Heart rate is widely applied to monitor an athlete during a training session. The linear relationship between heart rate and oxygen consumption is applied to determine the ideal training load. Several biochemical indicators are also applied by researchers to accurately reflect the training load; such indicators are blood lactate, urinary protein, and creatine kinase levels for determining the training intensity, urea and hemoglobin concentrations for judging the training volume, and the creatinine level for assessing the training result. In order to accurately monitor the training session and swiftly determine the training load, the paradigm has shifted from the cognitive experience of coaches and athletes to the scientific research of biochemical indicators in the athletes. Conducting sport training in a scientific manner is the key to enhancing the performance during competitions. ${ }^{(3)}$ Another important topic for an athlete is recovery from fatigue after an intensive training session. Sleep has been proved to have a close correlation with learning, cognition, the immune system, recovery from muscle fatigue, and the endocrine system. The quality of sleep can be reduced by muscle fatigue after a long intensive training, which in turn reduces the performance during the competition. ${ }^{(4)}$

\subsection{Psychological state of baseball athletes}

Mental breakdown is a syndrome that includes emotional exhaustion, depersonalization, and achievement reduction, which may occur in someone's life when the stress and pressure that an individual experience have increased to an extent they can no longer cope with. ${ }^{(5)}$ In the field of sport psychology, the phases of mental breakdown of an athlete are similar to those of a helping professional. However, unlike the helping professional, whose symptoms may be recognized by the surrounding people, athletes experiencing mental breakdown focus on competition events and training sessions. Excessive training and high expectations for favorable results of a competition are common. ${ }^{(6)}$ Most athletes have experienced sports injury. Physical therapy and recovery draw most of the attention after an injury, while psychological therapy is often neglected. The psychological stages an athlete may experience after a major sports injury are isolation, anger, compromise, loss of confidence, acceptance, surrender, and obedience. Also, it is common for an injured athlete to show fear of a second injury, which may lead to a reduced athletic skill, failure to concentrate, the rejection of the training, and increased exhaustion. The coach plays a crucial role during an athlete's recovery from a psychological disorder. Sincerity, mutual trust, and communication are the keys to helping an athlete regain confidence. ${ }^{(7)}$ The support of the sports psychologist and coach, and sports injury assistance are imperative for an athlete to reduce and recover from a psychological disorder. ${ }^{(8)}$ Because of the high unpredictability of incidents in sports, it is a common practice for a mental consultant to accompany the team during a competition event. During a routine training session, the sport psychologist helps athletes to strengthen their own mental health and better cope with anxiety during the competition. 


\subsection{Training environment of baseball athletes}

The Zuoying Training Center established in 1976 [renamed the National Sports Training Center (NSTC) in 2001] represents the commitment of the Taiwanese government to the development of sports. The NSTC has 37 acres available for track and field, swimming, and table tennis. An expansion in 1978 provided the capacity to host training for more than 20 types of sports. Several outstanding athletes have been trained at NSTC. However, substantial funding is required to renovate the current facility. ${ }^{(9)}$ As concluded from the need of NSTC for funding, we hypothesize that there are enormous improvement possibilities for training environments and facilities at the local college level. This research is aimed at clarifying the effect of the training environment on college baseball players.

\subsection{Research on the performance of baseball athletes}

An athlete's performance is often disrupted by sports injury, which is commonly caused by insufficient warmup, the lack of muscle strength, the imbalance of muscle strength, excessive training, the faulty or incorrect use of equipment, and difficult movements. Precaution is better than treatment; when a certain core muscle is being strengthened for one specific sport, it is also imperative to examine whether the antagonistic muscle is subjected to imbalance. ${ }^{(10)}$ The shoulder and elbow joints are two of the most vulnerable parts for a baseball player, and the most common injuries are muscle strain and muscle sprain. The reasons for injury include both internal and external factors. The internal factors include the athlete's psychological state, physical fitness, and skills, while external factors refer to the location, equipment, facilities, and environment. Precautions against injury include sufficient warm up, the reinforcement of safety measures (for instance, wearing protection gear and protective facilities at the training site), the monitoring of the athlete's condition, increasing the athlete's muscle strength and flexibility, and improving body coordination. In addition, creativity in athletic technique and the coach's coaching style are positively correlated with an athlete's performance, with creativity in technique being influenced by the coaching style. Hence, it is recommended that athletes search for a coaching style complementary to their own creativity. ${ }^{(11)}$

\subsection{Wearable device}

The wireless sensor network (WSN) technology has developed rapidly in recent years. The hardware design, manufacture, and protocols of WSN have all markedly advanced. Nevertheless, system developers are still facing many problems even as WSN systems are being used in practice. Besides the difficulties in setting up a WSN system, how to store the data acquired from sensors and how to provide a convenient method for users to access such data anytime, anywhere through the Internet, are also major problems. ${ }^{(12)}$ The shift to this data collection paradigm has created new challenges in indexing, navigating, documenting, and analyzing data. Furthermore, standard methods of analysis and quality assurance of sensorcollected data are lacking. Therefore, an automated system for checking the quality of and 
analyzing data on the basis of metadata would be tremendously advantageous for ecologists when they use sensor-collected data. To address the management and quality assurance problems plaguing sensor-collected data, we have developed a tool based on the Ecological Metadata Language (EML). This tool allows researchers to access EML, upload data, check data quality, and then run the $\mathrm{R}$ code on the server. Researchers can use the tool for data manipulation, calculation, and graphical display online without the need to install any statistical software. ${ }^{(13)}$ In the example of agricultural application, a wireless network sensor module is used to measure temperature data of crop growth layers inside a greenhouse and send the data to the back-end processor for further analysis. To analyze the data, the response surface methodology (RSM) in the statistical mode is used to determine the relationship between crop growth layers and temperature change, and then the temperature distribution status is shown using continuous 3-D images, providing the system manager with an immediate overview of how temperature is distributed dynamically inside a greenhouse, enabling him to assess and improve the efficiency of greenhouse environment control. The wireless wearable device was applied in the current research to structure the indicators for monitoring the development of players with respect to the three aforementioned factors during routine training.

\section{Research Methodology}

In this research, SPSS for Windows 20.0 was the platform used for statistical analysis. Descriptive statistics, independent sample t-test, single factor variance, and Pearson correlation analysis are the tools used in this research.

\subsection{Research instrument}

The questionnaire was divided into two parts. The first part concerns the personal information of the respondents. In the second part, players evaluate the correlation between their performance during the competition event and their physical fitness, psychological state, and training environment. The five-point Likert Scale is implemented as the measurement scale from " 1 = Strongly Disagree" to " 5 = Strongly Agree".

\subsection{Development of questionnaire}

There were four proposed factors in this research: physical fitness, psychological state, training environment, and competition event performance. In the questionnaire, the respondents were asked to deliberate their own opinion referring to different preset scenarios and then assign a score between 1 and 5 accordingly. The higher the score given by the respondents, the stronger the acknowledgement of the given scenario. After the questionnaires were collected, the statistical analysis was applied to the responses to test the significance of the proposed relationships. 


\subsection{Statistical analysis}

In this research, we used SPSS 20.0 to conduct the analyses: (1) descriptive analysis; (2) Student's t-test, and (3) Pearson correlation analysis. Descriptive analysis is a statistical approach to organizing, describing, and explaining the collected data. In descriptive analysis, the mass distribution is derived from the frequencies among different scales. By analyzing the distribution and percentage, a clear overview of the demographic information from the sample can be extrapolated. Student's t-test is applied to examine if the means of the two independent sets are significantly different from each other. Pearson correlation analysis explains the correlation between the competition event performance and the proposed factors, including physical fitness, psychological factor, and training environment.

\subsection{Unit of analysis}

This study focuses on college baseball players, who were asked to answer the questionnaire on the basis of their personal experiences. The Pingtung Meiho University baseball team in Taiwan was the sample for this study.

\subsection{Sampling methods}

Purposive sampling was conducted in the research. Since the designed questionnaire comprises forty questions, the number of questionnaires sent out in this study should be more than twice the number of questions in the questionnaire. In total, one hundred questionnaires were sent out and collected back; the effective response rate was $100 \%$. Although the measurement items were derived from previous studies, a pretest was conducted to verify whether the questionnaire delivered the intended message to the respondents. The final version of the questionnaire was prepared after the pretest.

\subsection{Scale measurement}

After the questionnaire was initially formatted, we consulted with the experts regarding the accuracy and acceptability of the questionnaire. Then, one hundred questionnaires were sent out. After the questionnaires were collected, first, the discriminant validity was analyzed to validate the collected questionnaire, followed by reliability analysis. The items with Cronbach's $\alpha$ of less than 0.7 were removed before the statistical analysis was conducted.

\subsection{Expert validity (face validity)}

After the questionnaire was initialized, three subject experts were consulted regarding the importance and applicability of the designed questions. The text readability and answerability of each question were reviewed and modified. No original proposed question was deleted after the consultation with the three subject experts. 


\subsection{Item analysis}

To examine the validity and reliability of the questionnaire, we separated the respondents' results into two groups on the basis of the summarized score of the following four designed factors: physical fitness, psychological state, training environment, and competition event performance. The first group, with the higher total score, represents $73 \%$ of the total respondents, while the second group, with the lower total score, represents $27 \%$ of the total respondents. After this group separation, the independent Student's t-test was performed to test the significance of the correlation among the physical fitness, psychological state, training environment, and competition event performance. When the p-value of Levene's test is significant at the level of 0.05 , the alternative hypothesis is validated. Next, Student's t-value was determined. In addition, the correlation analysis with the critical ratio (CR) was performed to examine the consistency across the questionnaire. If the $\mathrm{CR}$ value of a question is higher than 4 , the item discrimination is confirmed. After the analysis, all related coefficients were above 0.58 , which is higher than the standard of 0.30 .

\subsection{Exploratory factor analysis}

The aim of the exploratory factor analysis is to test the validity of the designed questionnaire. A thorough factor analysis of the entire questionnaire can be replaced with a direct factor analysis of each designed question under certain conditions, for example, a comprehensive literature study is conducted for the designed questionnaire to provide a clear definition of each factor and the opinions of experts are incorporated when finalizing the questionnaire. However, factor loading for each question should be higher than 0.4 during the factor analysis of each question. In the present study, we applied the principal component analysis (PCA) and Varimax method to extract underlying factors. The validity of the questionnaire is evaluated from the number of questions with eigenvalues higher than 1 proposed by Kaiser ${ }^{(14)}$ (i.e., Kaiser criterion) and factor loadings higher than 0.4 .

\subsection{Kaiser-Meyer-Olkin (KMO) value and Bartlett's sphericity test}

The KMO test is conducted prior to PCA in order to determine whether each designed factor is suitable for PCA. The results showed that the KMO values are $0.833,0.816,0.821$, and 0.834 across the physical fitness, psychological state, training environment, and competition event performance (i.e., local, national, or international, shown in Table 1), respectively, indicating

Table 1

Results of KMO analysis.

\begin{tabular}{lccc}
\hline Variable name & KMO & Bartlett's value & $p$-value \\
\hline Competition event performance & 0.834 & 1312.325 & $.000^{* *}$ \\
Physical fitness & 0.833 & 1306.235 & $.000^{* *}$ \\
Psychological state & 0.816 & 1298.334 & $.000^{* *}$ \\
Training environment & 0.821 & 1303.962 & $.000^{* *}$ \\
\hline **
\end{tabular}

${ }^{* *} p<.01$ 
that the data were appropriate for factor analysis. Furthermore, Bartlett's sphericity test showed that all the values are significant $(p<.001)$, indicating that there is covariance among the proposed factors. Therefore, the PCA is applicable to all the proposed factors.

As mentioned earlier, we applied the PCA combined with orthoblique rotation to extract the eigenvalue of each factor. For the data extraction factor, Kaiser criteria with an eigenvalue of 1.098 with the explanation of variance of $64.273 \%$ showed that the data included four factors matching the original version.

\subsection{Reliability analysis}

After examining the validity, the reliability test was conducted to examine the consistency and stability within the four proposed factors. Cronbach's $\alpha$ coefficient for the entire questionnaire reached 0.922 , while it was individually determined to be $0.727,0.802,0.786$, and 0.805 for the physical fitness, psychological state, training environment, and competition event performance (shown in Table 2). The results of the reliability test of the designed questionnaire were satisfactory.

\section{4. $\quad$ Research Results and Findings}

\subsection{Analysis of respondents' information}

The descriptive information obtained from the responses to the questionnaire is shown in Table 3.

\subsection{Analysis of physical fitness, psychological state, training environment, and competition event performance}

We applied the Pearson analysis to review the correlation among the physical fitness, psychological state, training environment, and competition event performance. The results are shown in Table 4. As can be seen, the significance was confirmed for each individual factor with all the other proposed factors; therefore, the relationships are all significant.

Table 2

Summary of validity of physical fitness, psychological state, training environment, and training performance as analytical factors.

\begin{tabular}{lll}
\hline Factor & Cronbach's $\alpha$ \\
\hline Physical fitness & 0.727 & \\
\hline Psychological state & 0.802 & \multirow{2}{*}{0.922} \\
\cline { 1 - 2 } Training environment & 0.786 & \\
\hline Competition event performance & 0.805 & \\
\hline
\end{tabular}


Table 3

Respondents' characteristics.

\begin{tabular}{lccc}
\hline Sex & Occurrence & Percentage & Accumulated percentage \\
\hline Male & 100 & 100.00 & 100.00 \\
Female & 0 & 0 & 0 \\
\hline Total & 100 & 100.00 & 100.00 \\
\hline Ever join championship & Occurrence & Percentage & Accumulated percentage \\
competition & 87 & 87.00 & 87.00 \\
\hline Yes & 13 & 13.00 & 13.00 \\
No & 100 & 100.00 & 100.00 \\
\hline Total & Occurrence & Percentage & Accumulated percentage \\
\hline Championship category & 18 & 18.00 & 18.00 \\
\hline Local & 76 & 76.00 & 76.00 \\
National & 6 & 6.00 & 6.00 \\
International & 100 & 100.00 & 100.00 \\
\hline Total & Occurrence & Percentage & Accumulated percentage \\
\hline Grade & 21 & 21.00 & 21.00 \\
\hline Freshman & 39 & 39.00 & 39.00 \\
Sophomore & 21 & 21.00 & 21.00 \\
Junior & 19 & 19.00 & 19.00 \\
Senior & 100 & 100.00 & 100.00 \\
\hline Total & 0 & Percentage & Accumulated percentage \\
\hline Experience in baseball & Occurrence & 0 & 0 \\
\hline$\leq 1$ year & 0 & 3.00 & 3.00 \\
2-4 years & 3 & 22.00 & 22.00 \\
5-7 years & 22 & 42.00 & 42.00 \\
8-10 years & 42 & 33.00 & 100.00 \\
ב10 years & 33 & & \\
\hline Total & & 100.00 & \\
\hline & & & \\
\hline
\end{tabular}

Table 4

Results of Pearson correlation analysis.

\begin{tabular}{|c|c|c|c|c|}
\hline \multirow{2}{*}{ Factor } & \multicolumn{4}{|c|}{ Factor } \\
\hline & (1) & (2) & (3) & (4) \\
\hline (1) Physical fitness & 1 & & & \\
\hline (2) Psychological state & $.556\left(^{* *}\right)$ & 1 & & \\
\hline (3) Training environment & $.416\left(^{* *}\right)$ & $.401\left(^{* *}\right)$ & 1 & \\
\hline $\begin{array}{l}\text { (4) Competition event } \\
\text { performance }\end{array}$ & $.465\left(^{* *}\right)$ & $\left..6322^{* *}\right)$ & $.386\left(^{* *}\right)$ & 1 \\
\hline
\end{tabular}

\section{Conclusion}

The objective of this research was to understand the correlation among the physical fitness, psychological state, training environment, and competition event performance by applying a rigorous scientific study. The meanings of statistical results are described below. In the first section, research conclusions are presented, and in the second section, we give some recommendations. 
The relationships raised in this research were tested using the software SPSS 20.0. The research conclusions are as follows.

\subsection{Correlation analysis among physical fitness, psychological state, training environment, and competition event performance}

The results of this research confirmed that significant correlations exist between each individual factor and all the other proposed factors. In other words, there is strong evidence of correlation among the physical fitness, psychological state, training environment, and competition event performance of athletes.

\subsection{Strategy of wearable device}

The wearable device has evolved rapidly since its debut on the commercial market, and today, it is widely applied in both leisure and medical activities. During competition events and training sessions, the wearable device can be used to collect real-time statistics from baseball athletes, such as the heart rate, blood pressure, muscle relaxation, blood oxygen concentration, and the speed of swing. The collected data is transmitted through the wireless network and received by coaches and sports injury therapists for further analysis of a player's physical and psychological status. As a result of such continuous monitoring, coaches are able to adjust their tactics during the competition or training to prevent the occurrence of sports injury.

Besides applications in physical and psychological analyses, the wearable device can also be used to monitor the conditions of the surrounding environment during the competition event, such as the humidity, temperature, brightness, wind direction, and wind strength. The collected data combined with big data analysis is helpful to coaches and athletes in making tactical decisions and predicting the strategies of opponents.

The data collected from the wearable device and the big data analysis can lead to the digitalization of the physical and psychological statuses of players during the training and competition. The collected and analyzed data are useful for building a customized behavior model for individual players and also pinpoint the potential risk of sports injury. Baseball players could utilize such models to adjust their posture and status, monitor fatigue and stress, reduce the chance of sports injury, and improve their competition event performance.

\subsection{Recommendation}

In this study, the Pingtung Meiho University baseball team was the research target. Meiho University is a private university: thus, we questioned whether differences in funding between public and private colleges play a role in the designed research. Moreover, the results of this study clearly supported the argument that, in addition to the player's physical fitness, coaches should pay greater attention to the player's psychological status as well as the training environment to attain a better training and competition performance. Finally, the question of whether significant variance exists with other factors will be incorporated in future studies. 


\section{Declaration of conflict interest}

The authors declare no potential conflicts of interest with respect to the research, authorship, and/or publication of this article.

\section{Funding}

The authors received no financial support for the research, authorship, and/or publication of this article.

\section{References}

1 S. S. Feng, Y. C. Chang, T.-Y. Wu, S.-Y. Fang, H. H. Ho, and S. C. Cheng: J. Exercise Health Res. 6 (2017) 25.

2 H. C. Chen, P. Y. Lee, H. L. Tai, and Y. F. Liou: Sports Exercise 17 (2015) 50. https://doi.org/10.5297/ ser.1701.005

3 C. F. Wu: Q. Chin. Phys. Educ. 11 (1997) 71. https://doi.org/10.6223/qcpe.1101.199706.1609

4 L. J. Chen, B. L. Hung, and S.-H. Fang: J. Nati. Taiwan Univ. Sport 5 (2016) 1. https://doi.org/10.3966/222653 5X2016060502001

5 L. Nowak: https://www.brightquest.com/blog/a-psychotic-break-vs-a-mental-breakdown-comparingsymptoms-and-treatment-options/ (accessed August 2020).

6 C. L. Nien and J. H. Lu: S. Res. Rev. 49 (2000) 45. https://doi.org/10.6162/srr.2000.49.14

7 K. H. Lai, Y.-W. Chang, and T. S. Chuang: J. Phys. Educ. Sport Sci. 2 (2016) 117. https://doi.org/10.6634/jpssccu.200406.02.12

8 C. M. Liao: Q. Chin. Phys. Educ. 8 (1994) 40. https://doi.org/10.6223/qcpe.0801.199406.1907

9 C. Y. Lin and C.Y. Fan: J. Phys. Educ. Natl. Taitung Univ. 16 (2012) 1. https://doi.org/10.29874/ JPENTU.201206.0001

10 C. J. Chiu and K. C. Chen: J. Phys. Educ. Sport Sci. 21 (2015) 23. https://doi.org/10.6634/jpss-ccu.201512.21.03.

11 C. S. Wu, M. H. Chen, and Y. C. Chen: Chung Yuan Phys. Educ. J. 3 (2013) 49. https://doi.org/10.6646/ cypej.2013.3.49

12 H. C. Yu and Y. T. Peng: CCL Tech. J. 123 (2008) 60. https://doi.org/10.29917/CCLTJ.200803.0010

13 C. C. Lin, C. W. Hsiao, S. S. Lu, and M.-R. Jeng: Taiwan J. For. Sci. 23 (2008) 279. https://doi.org/10.7075/ TJFS.200809.0279

14 H. F. Kaiser: Educ. Psychol. Meas. 20 (1960) 141. https://doi.org/10.1177/001316446002000116 